\title{
The Power of Pharmacological Sciences: The Example of Proton Pump Inhibitors
}

\author{
Reynold Spector $^{\mathrm{a}}$ Elliot S. Vesell ${ }^{\mathrm{b}}$ \\ ${ }^{a}$ Robert Wood Johnson Medical School, New Brunswick, N.J., and Harvard-MIT Program in the \\ Health Sciences, Cambridge, Mass., and ${ }^{\mathrm{b}}$ Department of Pharmacology, Pennsylvania State University \\ College of Medicine, Hershey, Pa., USA
}

\section{Key Words}

Proton pump inhibitors · Drug development - Strategy for drug discovery $\cdot$ Stomach acid

\begin{abstract}
Critics have questioned the foundational principles of pharmacological sciences and modern drug therapy; they also claim that drug therapy is often too expensive or of uncertain value. Contemporaneously, alternative medicine has bloomed. Yet the US government began to pay for drug therapy under Medicare in 2006, an explicit recognition of the value of modern drug therapy. To clarify this confusion, we review the philosophical and scientific foundations of pharmacology, drug discovery and development, the attendant strategies and successful results. We also review and answer the major attacks on the philosophical and scientific foundations of modern pharmacology and drug therapy. Finally, we define the characteristics of an ideal drug. As an example of the principles and strategies of modern pharmacological sciences and their successful application, we focus on the discovery and development of proton pump inhibitors (PPIs) of stomach acid production. This class of drugs approaches the ideal and exemplifies successful application of modern pharmacological principles to drug discovery and development. Moreover, the use of PPIs as a pharmacological tool allowed the resolution of important scientific questions, e.g., the role of stomach acid in peptic diseases of the stomach, duodenum and esophagus.
\end{abstract}

Copyright (C) 2006 S. Karger AG, Basel

\section{KARGER}

Fax +4161306 1234 E-Mail karger@karger.ch www.karger.com

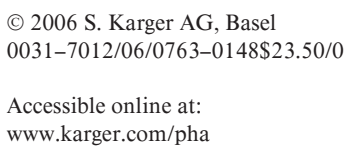

In the 20th century, medical scientists focused with great success on the treatment of the patient $[1,2]$. Moreover, they developed preventive strategies with proper nutrition, hormones, drugs and vaccines [1,2]. The disciplines of basic and clinical pharmacology (henceforth termed pharmacology) participated actively in these advances. However, in recent years critics have attacked both pharmacology and modern drug therapy [3, 4]. Prescription drugs have been characterized as excessively expensive and, in many cases, of uncertain value [3, 4]. As we and others have pointed out, these criticisms contain some truth [5-7]. Accordingly, for many drugs care should be taken to balance their risks and benefits.

In parallel with these criticisms of pharmacology and drug therapy, several non-traditional approaches to treatment and prevention of diseases have blossomed to fill the perceived void [4, 8]. Alternative medicine, including homeopathy, megavitamin therapy and acupuncture, has become popular and developed into big business [4, 6, 8], despite the fact that these modalities carry their own risks, in addition to uncertain benefits.

On the other hand, many experts and independent observers, including the US government, understand the value of pharmacology and drug therapy. Beginning in 2006, the US government will pay, in part, for drug therapy under Medicare. The government recognized that some new drugs can confer distinct medical benefits.

To clarify some of the confusion about what modern pharmacology and drug therapy can accomplish and why, we review briefly the very compelling but generally un-

Elliot S. Vesell, MD

Department of Pharmacology, Pennsylvania State University College of Medicine

500 University Drive

Hershey, PA 17033 (USA)

Tel. +1 717531 8285, Fax +1 717531 5013, E-Mail esv1@psu.edu 
Table 1. Therapy available for peptic ulcer disease in 1985 [1]

\section{Antacids}

$\mathrm{H}_{2}$-blockers (cimetidine, ranitidine)

Sucralfate

Colloidal bismuth

Surgery including partial gastrectomy and/or vagotomy

Psychiatric therapy including antidepressants

derappreciated philosophical and scientific foundations of pharmacology, drug discovery and development. We also examine attendant pharmacological strategies and several examples of successful results. We focus on the discovery and development of the proton pump inhibitors (PPIs) as an instructive example of pharmacology in theory and practice [9]. Not only did safe and effective treatments emerge for peptic ulcer disease, gastritis and esophagitis, but important scientific questions were answered definitively, e.g., what role does stomach acid play in causing these diseases? Concurrently, the very important discovery of the role of Helicobacter pylori in the pathophysiology of some cases of ulcer disease and gastritis (but not esophagitis) led to even greater therapeutic efficacy $[10,11]$.

\section{The Problem: Historical Background}

For millennia, gastritis, ulcer disease and esophagitis plagued mankind. By 1985, several causal hypotheses were advanced to explain these common, often serious upper gastrointestinal (UGI) diseases [1]. The most prominent hypothesis was that either excessive stomach acid production (e.g., in stress-induced ulceration) and/or inadequate protection against stomach acid were causally related to peptic ulceration, esophagitis and some types of gastritis in these patients [1].

In 1985, numerous therapies to treat and prevent peptic ulcer disease and gastritis existed (table 1) [1]. These medical therapies generally supported the notion of excess acid production (relative or absolute) by the stomach of such patients. Surgical therapy, which was often effective, also supported the excess acid hypothesis since the operations involved either partial removal of the stomach or vagus nerve ligation (to reduce acid production) or both. In 1985, the most common indications for surgical therapy of peptic ulcer disease were bleeding, perforation, obstruction or 'intractable' symptoms [1]. In the esophagus, surgery was usually reserved for patients with intrac- table symptoms or physical abnormalities (e.g., stricture). After the landmark discovery and development of the histamine receptor-2 blockers, exemplified by cimetidine, and then the PPIs and their widespread use, such surgical procedures were rarely required.

However, before introduction of the PPIs, there were some observers who challenged the predominant causal role of acid production by the stomach in the pathogenesis of esophagitis, gastritis and peptic ulcer disease. Some postulated psychiatric or nutritional causes [1]. Moreover, several discordant observations existed. First, shortterm (6-8 weeks) colloidal bismuth therapy seemed to confer longer-lasting benefit than cimetidine [11, 12]. Both cimetidine and bismuth therapy accelerated peptic ulcer healing comparably, but patients treated with bismuth had fewer recurrences when the therapy was discontinued [12]. In retrospect, this was almost certainly due to the activity of bismuth against $H$. pylori. Second and related to the beneficial colloidal bismuth effect, new reports of what are now recognized as $H$. pylori in the stomach appeared $[10,11]$. These bacteria, we currently believe, are contributory causes of gastritis and peptic ulcer disease (but not esophagitis) in some patients (see below). The discovery of the causal role played by $H$. $p y$ lori in peptic ulcer disease and gastritis appropriately culminated in the award of a Nobel Prize in 2005 [11].

A second piece of important new biology emerged in 1991 with the discovery of two distinct cyclooxygenase (COX) enzymes, designated COX-1 and COX-2 [13]. It is now established that inhibition of both COX-1 and COX-2 by nonspecific nonsteroidal anti-inflammatory drugs (NSAIDs) induces in some patients both symptomatic and asymptomatic gastritis and ulcerations in the stomach and duodenum [13]. Treatment of human subjects with specific COX-2 inhibitors (e.g., refocoxib) damages the UGI tract much less than dual inhibitors of COX-1 and COX-2 [13]. Clearly, COX-1 plays a constitutive role in producing prostaglandins that 'protect' the UGI tract of humans against gastritis and ulcer. COX-2 inhibitors are also effective NSAIDs [13].

Third, the PPIs helped clarify and diagnose certain previously obscure cases involving patients with chest pain, sore throat, laryngitis and asthma as described below.

At this point, we should define and clarify several important terms [1]. By disease, we mean scientifically verifiable abnormal processes in the patient's body [1]. By illness, we mean the patient's subjective feeling of not being well or normal [1]. By sickness, we mean the perception of disease or illness in an individual patient by outside observers. In a group of patients with objective (ob- 
servable by endoscopy or biopsy) gastritis, esophagitis, and/or peptic ulcers, an individual patient may have severe, moderate, mild or no symptoms. This observation that disease can occur with or without symptoms or conversely that symptoms can occur with or without disease has been repeatedly and systematically documented both in the community and in carefully controlled experiments in volunteers.

\section{Basic Philosophical Principles Underlying Medicine and Science}

In recent years, as noted above, serious challenges have been directed at certain clinical practices including the use of some drugs [6,14]. Critics have challenged not only the utility and safety of many forms of drug therapy and the perceived excessive costs, but also the foundational principles [3-6, 14]. As described in the rest of this paper, these challenges to the clinical use of many drugs have been met and overcome. The PPIs provide an excellent example. The scientific and medical justification for the widespread use of the PPIs as drugs is overwhelming. To appreciate the real value of this class of drugs, one should understand the epistemological assumptions and methods involved in the discovery and development of the PPIs. Moreover, this understanding justifies the vast resources used in the development of these drugs in thousands of patients.

Furthermore, practical reasons require understanding the basis and rationale for the development of PPIs or any drug for that matter. For example, the regulatory bodies in the USA (FDA), Europe and now the rest of the world justifiably demand that registration and sale of new drugs be based on sound medical science with trustworthy philosophical (especially epistemological) underpinnings. How did the PPI developers do this?

First, they employed the Standard Model of the World and our place in it (fig. 1) [15, 16]. In a general sense, all basic philosophy and science deal with interactions between the human subject, the world, and our representations of the world [15]. One of the principal objectives of medicine and all science is to find the truth. Clearly, truth depends on the accuracy of our representations of the world. More specifically, there are two general definitions of truth (table 2) [17]. In clinical pharmacology and medicine, acceptance of a statement as 'true' requires satisfaction of both definitions $[16,17]$.

Second, to obtain the truth, a series of assumptions must be made (table 3) [14-17]. An example of the as-

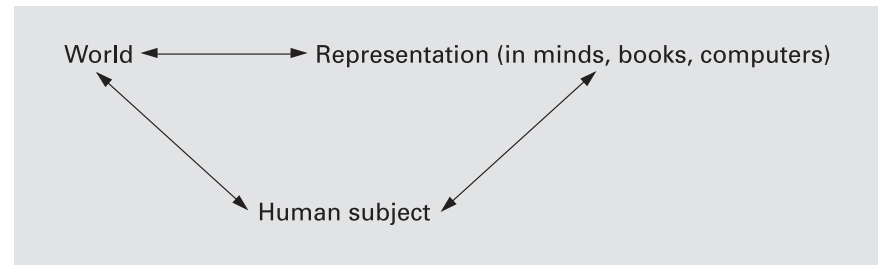

Fig. 1. Standard Model of the World - scientific realism [14, 15].

Table 2. Two definitions of truth in clinical medicine [17]

Realist definition (Quine): 'What is true is the sentence (statement or proposition), but its truth consists in the world's being as the sentence says' (i.e., corresponds to freestanding reality)

Pragmatic definition (Dewey): The truth is what 'works'. As part of the pragmatic definition and more broadly the scientific enterprise, the truth should be coherent with other knowledge, repeatable and, as the foundation of scientific hypotheses/principles, lead to correct predictions

Table 3. Assumptions underlying medical science [1, 14-19]

There exists a mind-independent world

The world is regular

Chance is random

Causal principles exist (Kant's Principle of Sufficient Reason, i.e., every event has a cause)

Scientific certainty is approachable

Truth, sincerity, and accuracy are valuable

Methods can be discovered to:

Adequately describe observed phenomena

Consistently explain observed phenomena Methods can be discovered to identify causes Inter-subjective observations in normal people match

sumption that chance is random is that a nonbiased coin will, if flipped repeatedly, come up heads half of the time. Moreover, in the last 150 years, we have recognized that information entering the brain from the outside world is filtered through our sense organs and then transformed by complex processes in the sense organs and brain into usable information. This is perhaps best understood with vision. Objects in the world are not colored per se. Rather they reflect certain wavelengths, transformed in the eye (by changes in the three color pigments) into electrical impulses, then routed to various regions in the brain for further processing. With knowledge of how our sense organs and brain handle information from the world and 
Table 4. Causality (history) $[1,15,18,19]$

Aristotle - material, formal, efficient, teleological causes

Newton - inertia

Hume - associations

Kant - imposition by brain

Necessary and sufficient causes

Koch's postulates (in infectious disease)

Contributory (precipitating, predisposing, sustaining) causes

Table 5. Evidential criteria for establishing causality (truth) in development of drugs $[1,5,6,16,19,20]$

Kuhn's general criteria: accuracy, consistency, scope, simplicity, fruitfulness

Medical criteria:

Satisfy both definitions in table 2

Evidence

Hypothesis testing with validated methods

Study one variable at a time

Dose-response relationships

Prospective controlled blinded randomized trials

(where necessary)

Multiple lines of evidence - same results

Correct predictions

Replication in multiple laboratories

Inter-observer agreement

Table 6. Challenges to the Standard Model Approach [15-19]

Hume - problems of induction

All As are B

Past predicts future

Kant's mental constructionism

Quine

Duhem thesis

Holism and under-determination

Nietzsche

Perspectivalism and post-modernism

Bad science, e.g., misleading epidemiology studies

confirmation by independent methods (e.g., machine outputs, microscopes, and photography) we can make increasingly accurate descriptions, explanations and models of diseases and treatments.

Third, the problem of causality is central to our understanding and treatment of diseases, e.g., what role does acid play in the pathophysiology of UGI disorders [15, 18]. To answer this question, one needs to understand and address the notion of causality, a very complex and difficult concept [15-18]. A cause is defined as "that factor which is possible or convenient for us to alter in order to produce or prevent an effect. This concept contains two components: production of an effect, and an understanding of its mechanisms' [16].

To understand causality, it is helpful to review briefly historical thinking about it (table 4). Aristotle believed that bodies in motion required constant force (efficient cause) to keep them moving; that the seed contained the adult (teleological cause) [14, 15, 18]. After more than 2,000 years, Newton overturned Aristotle in physics with the concept of inertia. Hume further advanced our understanding by postulating that our notion of causality depended on well-documented associations [15]. Kant believed the mind (brain) imposed notions of time, extension and causality on nature $[15,18,19]$. More recently, the concepts of necessary and sufficient cause and Koch's postulates were clearly delineated $[1,18,19]$. Finally, for many situations (e.g., high cholesterol as a cause of disease) the idea of contributory causality emerged [1]. This concept requires statistical thinking and its attendant science, since not everyone with the 'cause' develops the disease nor does everyone lacking the 'cause' fail to develop the disease. Because of the nature of contributory causality, large randomized controlled clinical trials are often required to establish the value of certain treatments, e.g., lowering serum cholesterol with drugs [20].

Finally, the types of evidence required to accept hypotheses in pharmacology and medicine as correct, either as explanations or even 'true' causal laws, have become clearer in recent years (table 5) [1, 14-20]. These are especially important in situations where there is contributory causality - less so in situations where necessary and sufficient causal agents are being studied.

\section{Challenges to the Standard Model and Assumptions}

In the last three centuries, important challenges were raised to the Standard Model (fig. 1), assumptions (table 3 ), assessments of causality (table 4), and the justification of beliefs based even on 'strong' evidence (table 5). In retrospect, these challenges (table 6) were generally helpful and forced even stronger evidentiary standards.

Hume, for example, pointed out that one can never say 'All As are B', unless all As have been observed. The one black swan disproves the statement that 'All swans are white'. More importantly, Hume argued that knowledge of the past does not guarantee the future (the problem of 
induction) $[15,18]$. The problem of induction cannot be entirely overcome - only minimized by large quantities of good data confirming a clear hypothesis and rejecting (often by falsification) competing hypotheses. Second, Kant was only partially correct that certain concepts (e.g., causality, time, extension-in-space) are innate and minddependent, and 'imposed on the world' by the structure and physiology of the mind/brain [15, 18]. However, Kant was correct that information (sensory) is modified by human perceptual and conceptual systems. We now recognize that with proper interpretation, the use of instruments and other appropriate methods and techniques, we can often closely approach 'seeing' and understanding mind-independent reality. Modern science has, to a great extent, revealed much more than the 'shadows' of Plato's cave [14-19].

Quine also raised several important issues including the notion of holism, under-determination of theories by evidence, and, as Duhem pointed out a century ago, the all-too-common difficulty in accepting one theory as the 'best' one $[14,15,19]$. Many of these objections are important to consider in sound medical science (table 6). For example, scientific holism notes that one 'established theory' depends in turn on a whole chain of data, assumptions and other theories any of which, if incorrect, would undermine the 'established theory'. The solution to these objections is to perform replicate studies and utilize multiple independent methods. If data from such studies all produce the same or consistent results, one can provisionally accept the tested hypothesis. For example, there are at least six different methods to measure the acidity of stomach contents (methods based on alkali titration, membrane permeability, dyes, ion exchange, etc.), all of which yield the same results. Such overwhelming data with different validated methods employing proper internal and external standards in laboratories all over the world are extremely convincing even to skeptics.

Nietzsche and certain postmodernists have criticized the humanities, as well as the social and medical sciences, for exaggerating claims to truthfulness and utility [6]. These criticisms outside medical and physical sciences have substantial merit, but are irrelevant to the current discussion and hence can be ignored. For example, a $\mathrm{pH}$ meter is not influenced by considerations of political power, social position or nuances of language.

Especially harmful to the proper treatment and prevention of disease is the large amount of bad science that has been and continues to be performed, disseminated and used for marketing therapy (e.g., epidemiological studies of estrogen in postmenopausal women and mega-
Table 7. Scientific questions in 1985

What is the role of stomach acid in NSAID-induced gastritis and GI ulceration?

What is the role of stomach acid in the Zollinger-Ellison syndrome? Is overproduction of acid the sole 'cause'?

What is the role of stomach acid in 'stress ulceration'?

What is the role of stomach acid in DU, GU, gastritis and esophagitis in those not infected with $H$. pylori?

What is the role of stomach acid in DU, GU, gastritis and esophagitis in those infected with $H$. pylori?

GI = Gastrointestinal; DU = duodenal ulcer; GU = gastric ulcer.

vitamin $\mathrm{E}$ for cardiovascular risk protection) $[6,16,17]$. Demonstrated to be erroneous, such studies require repudiation since bad medical 'science' indirectly harms all medical science and many patients by propagating falsehoods.

In summary, there exist numerous veridical philosophical and scientific approaches, methods and criteria. These approaches, methods and criteria are widely accepted both by scientists and regulatory authorities - the overriding objective being the pursuit of truth, especially about hypotheses. The discovery, development and use of the PPIs exemplify the use of these principles in finding a way to help patients with UGI diseases as described below. These methods also allowed validation of the role stomach acid plays in UGI diseases.

\section{The Challenge}

In 1985, the treatment and prevention of peptic ulcer disease, gastritis and, to a lesser extent, esophagitis (table 1), although improved from 20 years before, remained far from ideal [1]. Medical treatments available in 1985 for patients did not uniformly relieve UGI symptoms, signs, and complications (perforation, obstruction, bleeding and intractability). Surgery was invasive, expensive and not ideal. Simpler operations (e.g., selective vagotomy) were not uniformly successful. Also, a series of critical scientific questions were unanswered since, in patients, one could not sufficiently 'block' stomach acid production or its effects. Some of these questions are enumerated in table 7. Thus, in 1985 there was a large opportunity for improving the therapy of UGI diseases. 


\section{Pharmacology, Discovery and PPI Development}

As noted above, a leading hypothesis considered acid production by the stomach of patients not just a contributory, but a necessary cause of all conditions listed in table 7. To answer that question, one would probably need to block completely (or neutralize) stomach acid production in these patients around the clock. Was this possible? Would it be safe?

An analogous question, raised several decades before, concerned the cholesterol hypothesis [20]: did excess cholesterol cause atherosclerosis and heart disease? One approach was to block the final step in cholesterol biosynthesis. This was accomplished with the development of MER-29. Unfortunately, build-up of the penultimate product, desmosterol, proved toxic in patients and MER29 was withdrawn. Fortunately, inhibitors of an earlier, rate-limiting step in cholesterol biosynthesis (HMG-CoA reductase) finally led to the statins, safe and effective drugs [20]. This work demonstrated that it was neither necessary nor safe to block cholesterol biosynthesis completely, but rather that normalization of excessive cholesterol concentrations was therapeutically advantageous.

The strategy taken by the discoverers of the first PPI, omeprazole, was bold in view of the experience with MER-29. They postulated that the best and perhaps only way to block stomach acid secretion completely would be to target the final step in stomach acid secretion, the enzyme $\mathrm{H}^{+}, \mathrm{K}^{+}$-ATPase, with a specific, potent inhibitor [9]. Only then could one test the hypotheses and questions in table 7 with a PPI as a tool. In 1985, it was recognized that blockade of two of the three main stimulants to acid production (histamine and acetylcholine), although helpful, did not completely halt acid production in patients, especially after eating $[9,21]$. An inhibitor of gastrin, the third principal stimulant to acid production, was never developed. However, one theoretical concern (to the development of PPIs) was raised repeatedly: would complete blockade of acid production by inhibiting $\mathrm{H}^{+}, \mathrm{K}^{+}-$ ATPase have harmful consequences on the stomach itself [9]? For example, many scientists and regulators were concerned that the resultant lack of feedback inhibition of gastrin production by the stomach (by acid) would lead to hypergastrinemia and enterochromaffin-like hyperplasia or tumor formation analogous to the situation with MER-29. Unlike rats, these safety concerns proved insignificant in humans [9].

Fortunately, the discoverers of omeprazole pressed ahead with their efforts and made a potent, specific in- hibitor of the human stomach $\mathrm{H}^{+}, \mathrm{K}^{+}$-ATPase. Subsequently other PPIs were developed that are similar to omeprazole in terms of structure, mechanism of action, pharmacokinetics, efficacy and safety [21, 22]. These drugs all have a sulfinyl group between substituted benzimidazole and pyridine rings. After diffusion from the blood into the acid environment of the stomach secretory canaliculi, the PPIs form a sulfenamide which irreversibly inactivates the ATPase $[9,21,22]$. This phenomenon allowed for once-a-day dosing in most patients [9, 21]. The developers of the various PPIs also overcame several other formidable technical problems. For example, the PPIs are acid-labile so that before absorption in the intestinal tract, the drug had to be protected from stomach acid with acid-resistant coatings [9, 21].

\section{Consequences of PPI Investigations and Use}

Several questions were answered with the availability of safe and potent, specific, irreversible inhibitors of $\mathrm{H}^{+}, \mathrm{K}^{+}$-ATPase that permit essentially $90-100 \%$ inhibition of stomach acid production around the clock in compliant patients receiving adequate doses $[9,21,22]$. Demonstrated even in the initial clinical trials, inhibition of acid production by PPIs led to rapid healing, symptomatic relief and near elimination of complications in patients with peptic diseases of the stomach and duodenum even in the face of continued $H$. pylori infection, an exceptional result. The PPIs also healed esophagitis and esophageal ulceration $[21,23]$. These clinical studies were performed with careful measurement of symptoms, signs (e.g., endoscopic visualization and biopsy of ulcers and inflammation), and recording of complications (e.g., perforation, bleeding, obstruction, and stricture). Moreover, as far as is known in patients on clinical doses of PPIs, the primary action of the PPIs (in healing ulcers and gastrointestinal inflammation and controlling symptoms) is to inhibit stomach acid production [9]. This effect is consistent with the remarkable biochemical specificity, selectivity and the high potency of these drugs $[9,21]$. Of course, there are measurable secondary consequences, e.g., changes in gastric emptying, due to decreased acid secretion. These clinical data satisfied the evidentiary criteria in table 5 and challenges to believing the results and their interpretation (table 6). These clinical data also allowed registration and sale of the first PPI omeprazole in 1988 [9].

Over time, multiple clinical trials with the various PPIs expanded our knowledge base and answered conclu- 
sively the questions in table 7 , since we can now block acid secretion in these patients (with adequate doses of many PPIs) around the clock. First, PPIs as a class treat and prevent NSAID-induced UGI inflammation and ulceration and several are approved by the FDA for this use $[21,24]$. Second, PPIs protect the UGI tract against gastrin-induced ulceration in the Zollinger-Ellison syndrome, although high doses may be necessary to counter the intense gastrin-induced tendency to ulceration [21]. The Zollinger-Ellison syndrome consists of a triad comprising intractable, sometimes fulminating, often atypical gastric (or duodenal) ulcers, extreme gastric acidity, and finally gastrin-secreting tumors. Third, PPIs protect against stress-induced UGI ulceration, inflammation and bleeding (e.g., in the hospital) $[9,21]$. In these three cases, PPIs also help heal extant UGI inflammation and ulceration $[21,22]$. Fourth and fifth, in patients with or without $H$. pylori infection, PPIs promoted ulcer healing and either cured or markedly ameliorated UGI inflammation [9, 21, 22]. Of course, in patients with $H$. pylori infection, the bacteria should be eliminated with an antibiotic regimen containing a PPI to prevent recurrence $[9,21]$.

Based on these studies of inhibition of acid production, it became clear that stomach acid is more than just a contributory cause of stomach and esophageal ulcers, esophagitis and gastritis. Rather, stomach acid is a necessary cause; without acid there is little or no (peptic) gastritis, esophagitis or UGI ulceration. Without acid H. pylori does not cause peptic ulcers and UGI inflammation; even in the face of continued infection, ulcers will heal if adequate doses of PPIs are given [22]. In fact, even with stomach acid, many people harbor $H$. pylori without symptoms or apparent consequences. Thus, $H$. pylori is an important contributory cause of ulceration and inflammation in the stomach and duodenum in some individuals. (Obviously in diseased patients, we cannot overemphasize the importance of eradication of $H$. pylori if present.) Unlike in the stomach and duodenum, esophageal inflammation and ulceration are not caused by $H$. pylori but by stomach acid reflux [21, 23]. Gastritis, esophagitis and UGI ulceration caused by other agents such as viruses or fungi are clearly not relevant to this discussion.

Third, in addition to their therapeutic effect, the PPIs have yielded important diagnostic information. Some previously mysterious cases in which stomach acid was implicated (e.g., certain cases of laryngitis, asthma, and pharyngitis) have been clarified by the use of PPIs in such patients [21, 25, 26]. Apparently, in these patients, enough stomach acid is refluxed up the esophagus to damage the
Table 8. Properties of an ideal drug [2, 5]

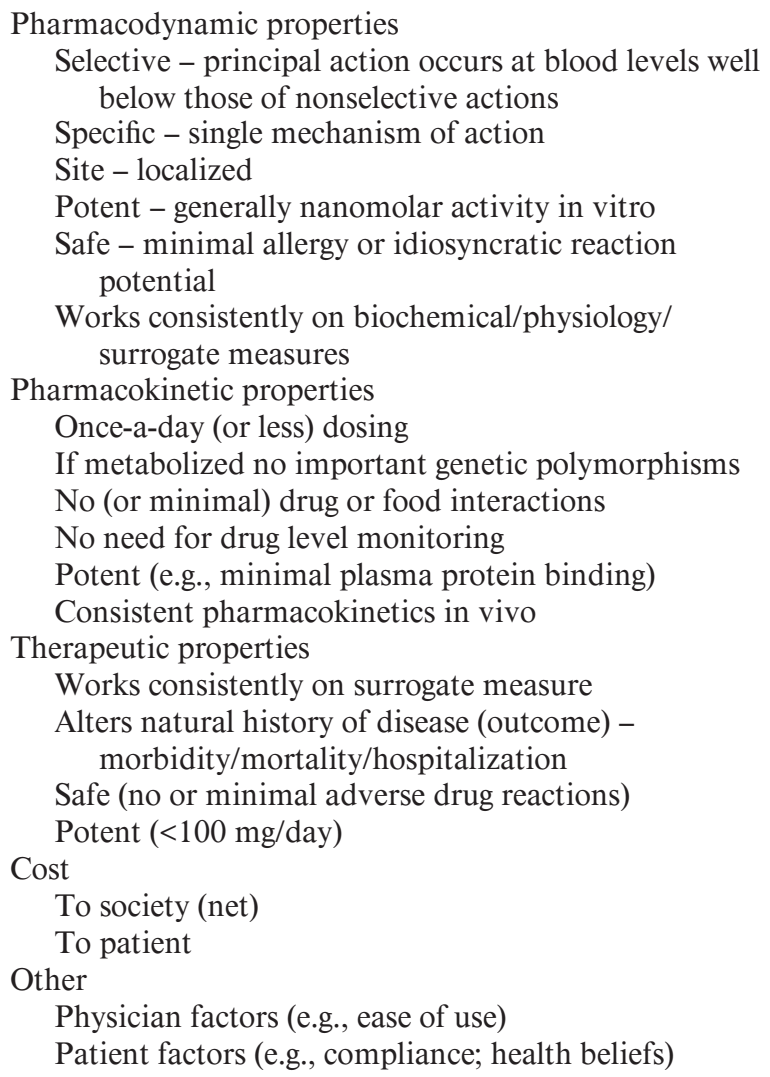

throat and then aspirated into the respiratory tract. Inhibition of stomach acid production minimizes the acidinduced damage to the throat and respiratory tract. Similarly, certain non-cardiac chest pain syndromes are eliminated by PPIs, thus establishing their UGI (probably esophageal) origin $[25,26]$.

Finally, as a note of caution, PPIs cannot cure malignant GI ulcers, nor can they eliminate lower esophageal sphincter dysfunction that allows reflux. Ulcers due to ingestion of corrosives (e.g., alkali) and toxins are obviously unrelated to the issues discussed herein.

Over time, a key question became: how close are the PPIs to ideal drugs for the treatment and prevention of duodenal ulcer, gastric ulcer, gastritis and esophagitis? Criteria for an ideal drug are listed in table $8[2,5]$. We now recognize that PPIs satisfy all of the criteria for ideal drugs (with the exception of rare clinically relevant drug interactions [21]), especially since the cost of the first of these drugs, omeprazole (at least in the USA), has become reasonable. In 2002, it became generic and sold over the counter. 


\section{Conclusion}

The PPIs represent one of the most significant advances in the treatment of UGI disorders. Discovery and development of the PPIs exemplify a rational approach employing sound philosophical, scientific and medical reasoning, use of the best techniques of biochemistry, molecular biology, and especially pharmacology and clinical development: a vast undertaking. The discoverers of the PPIs chose a sound strategy and painstakingly selected the correct enzyme target, the $\mathrm{H}^{+}, \mathrm{K}^{+}$-ATPase proton pump. We now know how to cause ulcers, gastritis and esophagitis, as well as how to remove them. We also understand the role of stomach acid and H. pylori. We can, in a sense, meet the ultimate philosophical criterion that, if you really understand something, you can build it. The consequences of this achievement include the tremendous benefit for millions of sufferers. Not only are the symptoms relieved and the disease ameliorated or eliminated, but hospitalization of these patients for complications, as well as deaths due to hemorrhage, perforation and obstruction, can be largely eliminated. Moreover, surgical intervention is rarely required. Accordingly, the PPIs constitute a compelling example of the successful application of pharmacological sciences to major medical and scientific problems.

\section{Acknowledgements}

The authors wish to thank Jennifer W. Schwing and Michiko Spector for their aid in research and preparation of the manuscript.

\section{References}

1 Spector R: The Scientific Basis of Clinical Pharmacology: Principals and Examples. Boston, Little, Brown, 1986.

$\checkmark 2$ Spector R: Progress in the search for ideal drugs. Pharmacology 2002;64:1-7.

$\checkmark 3$ Davidoff F: The heartbreak of drug pricing. Ann Intern Med 2001;134:1068-1071.

4 Trudeau K: Natural Cures 'They' Don't Want You to Know About. New York, Alliance, 2005.

$\checkmark 5$ Spector R, Vesell ES: A rational approach to the selection of drugs for clinical practice. Pharmacology 2002;65:57-61.

-6 Spector R, Vesell ES: Which studies of therapy merit credence? Vitamin $\mathrm{E}$ and estrogen as cautionary examples. J Clin Pharmacol 2002; 42:955-962.

7 Woloshin S, Swartz LM, Tremmel J, Welch HG: Direct-to-consumer advertisements for prescription drugs: what are Americans being sold? Lancet 2001;358:1141-1146.

$>8$ Eisenberg DM, Kessler RC, Foster C, Norlock FE, Calbins DR, Delbanco TL: Unconventional medicine in the United States - prevalence, costs, patterns of use. N Engl J Med 1993;328: 246-252.

9 Olbe L, Carlsson E, Lindberg P: A protonpump inhibitor expedition: the case histories of omeprazole and esomeprazole. Nat Rev Drug Discov 2003;2:132-139.

10 Marshall B, Warren J: Unidentified curved bacilli in the stomach of patients with gastritis and peptic ulceration. Lancet 1984;i:13111313.
11 Altman LK: A Scientist gazing toward Stockholm, ponders 'what if?'. New York Times 2005;Dec 6:F5.

12 Martin DF, Hollanders D, May SJ, Ravenscroft MM, Tweedle Dt, Miller JP: Difference in relapse rates of duodenal ulcer after healing with cimetidine or tripotassium dicitrato bismuthate. Lancet 1981;i:7-10.

13 Bombardier C, Laine L, Reicin A, Shapiro C, Burgos-Vargas R, Davis B, Day R, Ferraz MB, Hawkey CJ, Hochberg MC, Kvien TK, Schnitzer TJ: Comparison of upper gastrointestinal toxicity of rofecoxib and naproxen in patients with rheumatoid arthritis. VIGOR Study Group. N Engl J Med 2000;343:1520 1528.

14 Haack S: Defending Science - Within Reason Amherst, Prometheus, 2003.

15 Danto AC: Connections to the World. Berkeley, University of California Press, 1997.

16 Jenicek M: Foundations of Evidence-Based Medicine. Boca Raton, Parthenon, 2003.

17 Spector R, Vesell ES: The pursuit of truth: role of epidemiology/observation studies. J Clin Pharmacol 2000;40:1205-1210.

18 Sosa E, Tooley M (eds): Causation. Oxford, Oxford University Press, 1993.

19 Kilcher P: The Advancement of Science. New York, Oxford University Press, 1993.
20 Scandinavian Simvistatin Survival Study Group: Randomized trial of cholesterol lowering in 4,444 patients with coronary heart disease: the Scandinavian Simvistatin Survival Study (4S). Lancet 1994;344:1383-1389.

21 Hoogerwerf WA, Pasricha PJ: Pharmacotherapy of gastric acidity, peptic ulcers and gastroesophageal reflux disease; in Brunton LL (ed): Goodman and Gilman's The Pharmacological Basis of Therapeutics, ed 11. New York, McGraw-Hill, 2005, pp 967-981.

22 Thomson ABR: Are the orally administered proton pump inhibitors equivalent? A comparison of lansoprazole, omeprazole, pantoprazole and rabeprazole. Curr Gastroenterol Rep 2000;2:482-483.

23 Dent J, Brun J, Fendrick AM, et al: An evidence-based appraisal of reflux disease management: the General Workshop Group. Gut 1999;44(suppl 2):S1-S16.

24 Scarpignato C, Pelosini J: Prevention and treatment of non-steroidal anti-inflammatory drug-induced gastro-duodenal damage: rationale for the use of antisecretory compounds. Ital J Gastroenterol Hepatol 1999;31(suppl 1): S63-S72.

25 El-Serag HB, Lee P, Buchner A, Inadomi JM, Gavin M, McCarthy DM: Lansoprazole treatment of patients with chronic idiopathic laryngitis: a placebo-controlled trial. Am J Gastroenterol 2001;96:979-983.

-26 Ofman JJ, Gralnek IM, Udani J, Fennerty MB, Fass R: The cost-effectiveness of the omeprazole test in patients with noncardiac chest pain. Am J Med 1999;107:219-227. 


\section{Editorial Comment}

\section{Philosophic Traditions and Modern Pharmacology}

Melvin L. Billingsley, Editor

The recent spate of contentious and acrimonious issues surrounding drug safety, drug pricing and new drug development has led to more widespread public questioning of the rationale behind the use of modern therapeutic agents. Indeed, public trust in the established pharmaceutical enterprise is at a low point. In the accompanying commentary by Spector and Vesell, the authors lay out some of the philosophic issues which underscore the basis of modern pharmacology, and offer insight into how new drug development has led to agents which not only address vexing issues of disease, but also help elucidate some of the underlying causal pathologic elements, using the development of proton pump inhibitors as an example. As we enter the era of 'personalized' medicine and all of the potential to tailor-make drug therapies for specific targets and specific individuals, it becomes incumbent on pharmacologists to articulate the rationale and advantages afforded by some of the best current drug therapies. It is hoped that this commentary will provoke thought, encourage others to submit related commentaries and promote a healthy dialog, leading to even more exciting therapeutic developments in the future. 